\title{
Enrichment and purification process of astragalosides and their anti-human gastric cancer MKN-74 cell proliferation effect
}

\author{
Yang OuYang*, JianHua Huang, Zing'Ting OuYang, Jin Kang
}

Department of General Surgery 8W, Xiangya Hospital of Central-South University, Changsha, 410008 China

\begin{abstract} effects. line MKN-74. total astragalosides. showed a dose-and time-dependence. of MKN-74 cells.

Key words: Total astragalosides, DA201 Resin, MKN-74 Cell

\section{Introduction}

Radix Astragali is the dried root of perennial herbaceous plants Astragalus membranaceus (Fisch.) Bunge var. mongholicus (Bunge) Hsiao or A. membranaceus (Fisch.) Bunge of family Leguminosae. ${ }^{1}$ It mainly contains saponins, polysaccharides, flavonoids, amino acids and other chemical constituents, ${ }^{2-3}$ of whichtotal astragalosides have immunomodulatory, anti-viral, hepatoprotective, and gastric mucosa protective effects. ${ }^{4-5}$ In this paper, process conditions for extraction, purification and enrichment of total astragalosides by macroporous adsorption resin were investigated, and the inhibitory effect of total astragalosides on growth of human gastric cancer cell line MKN-74 was studied.
\end{abstract}

Background: Radix astragali mainly contains saponins, polysaccharides, flavonoids, amino acids and other chemical constituents of which total astragalosides have immunomodulatory, anti-viral, hepatoprotective, and gastric mucosa protective

Objective: To investigate the process conditions for extraction, purification and enrichment of total astragalosides by macroporous adsorption resin, and to study the inhibitory effect of total astragalosides on growth of human gastric cancer cell

Methods: UV spectrophotometry was applied to determine the adsorption and desorption capacity of macroporous adsorption resin on total astragaloside content, MTT assay was used to determine the inhibition of MKN-74 cell growth by

Results: The dynamic adsorption performance of DA201 adsorption resin was examined, and the dynamic adsorption curve of total astragalosides on DA201 resin column was plotted. Meanwhile, eluent and elution flow rate were investigated, the results showed that the choice of eluent of $80 \%$ ethanol, and a flow rate of $5 \mathrm{BV} / \mathrm{h}$ could maximize the yield of total astragalosides. MTT assay found that astragalosides could relatively pronouncedly inhibit the proliferation of MKN-74 cells, and the inhibitory effect was enhanced with the increase of astragaloside dose and the extension of processing time, which

Conclusion: DA201 resin can effectively enrich total astragalosides, total astragalosides have an inhibitory effect on growth

African Health Sciences 2014;14(1): 22-27 http://dx.doi.org/10.4314/ahs.v14i1.5

\section{Materials}

Reagents and drugs

Radix Astragali extract, self-prepared, containing $51.37 \%$ of total astragalosides; human gastric cancer cell line MKN-74, purchased from the Cell Bank of Beijing Institute of Cell Biology, CAS. DMSO, MTT (Sigma, USA), fetal calf serum (Gibco), RPMI 1640 medium (Invitrogen).

\section{Instruments}

Model 680 microplate reader (Bio-Rad, USA); General TV-1221 UV-vis spectrophotometer (General, Beijing); $\mathrm{CO}_{2}$ incubator (model CO-150, NBS, USA); SW-CJ-SF clean bench (Suzhou Purification Equipment Factory, Sujing Group).

\section{Methods}

Pretreatment of resin

Newly purchased DA201 and D101 macroporous resins were soaked in 2 column bed volumes (BV) of 95\% ethanol for $24 \mathrm{~h}$, respectively; after fully swollen, the resins were eluted with ethanol, and then with distilled water until there was no smell of ethanol and set aside. 


\section{Preparation of sample solution}

Radix Astragali extract was weighed and placed in the beaker, dissolved by addition of appropriate amount of distilled water ultrasonically for $10 \mathrm{~min}$, and then filtered to give the sample solution.

\section{Method for content determination}

$8 \mathrm{mg}$ of astragaloside reference substance was accurately weighed, dissolved in anhydrous ethanol to make the volume $50 \mathrm{~mL} .0,0.2,0.4,0.6,0.8$ and $1.0 \mathrm{~mL}$ of astragaloside reference substance solutions were accurately aspirated, evaporated to dryness in water bath, added with $0.2 \mathrm{~mL}$ of $5 \%$ vanillin solution and $0.8 \mathrm{~mL}$ of perchloric acid, heated in water bath for 20 min and then removed, cooled to room temperature, added with glacial acetic acid till the volume was $5 \mathrm{~mL}$, respectively, absorbance was then measured at $560 \mathrm{~nm}$ with $70 \%$ ethanol as blank control. Standard curve was plotted with concentration of reference substance on the $\mathrm{X}$-axis and absorbance on the $\mathrm{Y}$-axis, linear regression equation was obtained as $\mathrm{Y}=0.0312 \mathrm{X}-0.0145, \mathrm{R} 2=0.9996$.

\section{Determination of resin model}

Static adsorption effects of various types of resins were determined with adsorption capacity of astragalosides as the evaluation indicator. $2 \mathrm{~g}$ of pretreated DA201, D101 and AB-8 macroporous adsorption resins were taken, respectively, added with excess Radix Astragali sample solution and shaken at room temperature for $48 \mathrm{~h}$.

\section{Study of dynamic adsorption effect}

$10 \mathrm{~mL}$ of pretreated resin was taken and loaded on the column by wet packing method, Radix Astragali extract (mass concentration of total astragalosides of $0.85 \mathrm{mg} /$ $\mathrm{ml}$ ) was passed through the chromatographic column at a flow rate of $2 \mathrm{BV} / \mathrm{h}$, astragaloside content in the effluent was measured when $1,2,3,4,5,6,7,8,9,10$, $11,12,13$ and 14 fold volumes of samples were added, respectively, until the resin adsorption was saturated, dynamic adsorption curve of total astragalosides on DA201 resin column was plotted.

\section{Effect of flow rate on adsorption}

10 BV extract was passed through the chromatographic column containing $10 \mathrm{~mL}$ of resin at different flow rates, total astragaloside content in the effluent was detected, and the effect of flow rate on resin adsorption capacity was investigated.

\section{Selection of eluent}

$1 \mathrm{~g}$ of saturated adsorption resin was taken in 6 replicates, and water, $10 \%, 30 \%, 50 \%, 70 \%$ and $90 \%$ ethanol were taken as the eluent, respectively, flow rate was 5 $\mathrm{BV} / \mathrm{h}$. Content of total astragalosides was determined, and the elution effects at different ethanol concentrations were investigated.

\section{Inbibition of human gastric cancer MKN-74 cell growth by total astragalosides \\ Cell cultivation}

MKN45 cell lines were cultured in 10\% fetal calf serum-containing RPMI 1640 medium, and routinely subcultured in a $37 \square, 5 \% \mathrm{CO}_{2}$ incubator, cells in the logarithmic growth phase were collected and set aside.

Detection of the effect of astragelosides on cell proliferation by MTT assay ${ }^{6-7}$

Well grown cells in the exponential growth phase were taken, digested with $0.25 \%$ trypsin, and then prepared into cell suspension with a concentration of $1 \times 10^{5}$ cells $/ \mathrm{mL}$, and seeded into 96 -well plates at $1 \times 10^{4}$ cells per well, the MKN-74 cells were then treated with different concentrations of total astragelosides $(5,10,20$ umol/L), on the 3rd day, $20 \mu \mathrm{L}$ of MT'T solution was added to each well, and the incubation was continued for an additional $4 \mathrm{~h}$. Supernatant was removed carefully, each well was added with $150 \mu \mathrm{L}$ of DMSO, and shaken for $10 \mathrm{~min}$, absorbance (A) of each well was measured at $570 \mathrm{~nm}$ wavelength on automatic microplate spectrophotometer.

Inhibition rate $=$ (A value of negative control group A value of experimental group) / A value of negative control group $\times 100 \%$.

\section{Results}

\section{Result of resin model}

After full adsorption, adsorption capacity was calculated based on the absorbance before and after the adsorption, thereby confirming that DA201 adsorption resin had more superior adsorption performance (in Table1). 
Table 1: Results for determination of static saturated adsorption capacity of various resins

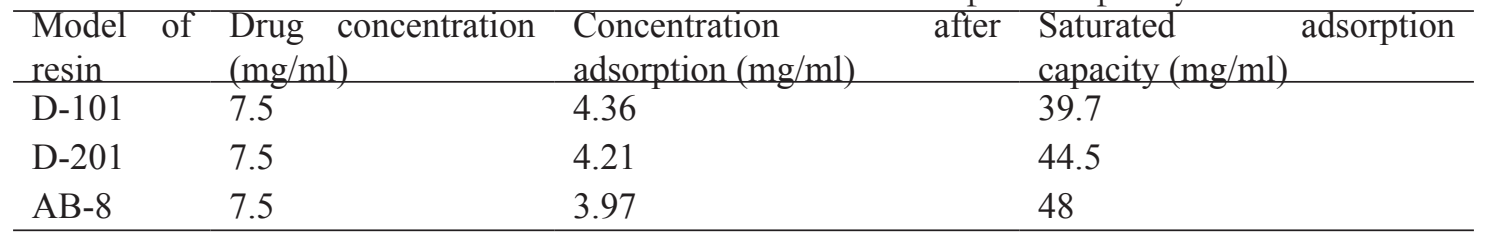

\section{Result of dynamic adsorption effect}

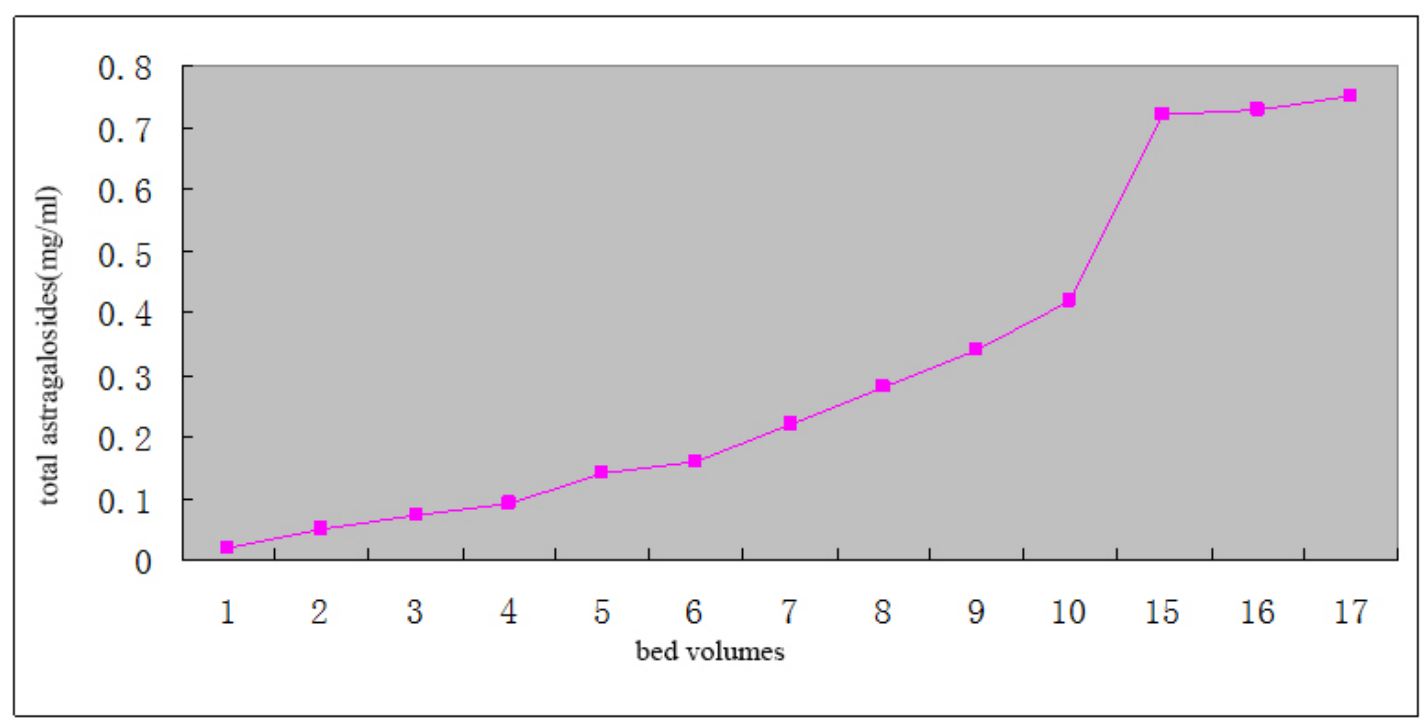

Figure 1: Dynamic adsorption curve of total astragalosides

As can be seen from Fig. 1, total astragalosides had relatively large leakage starting from $10 \mathrm{BV}$; mass concentration of total astragalosides was rising with the increase in the number of fractions, after rising to 15 $\mathrm{BV}$, the curve tended to flatten, which meant that the dynamic adsorption of DA201 resin on total astragalosides tended to saturation.

\section{Result of flow rate on adsorption}

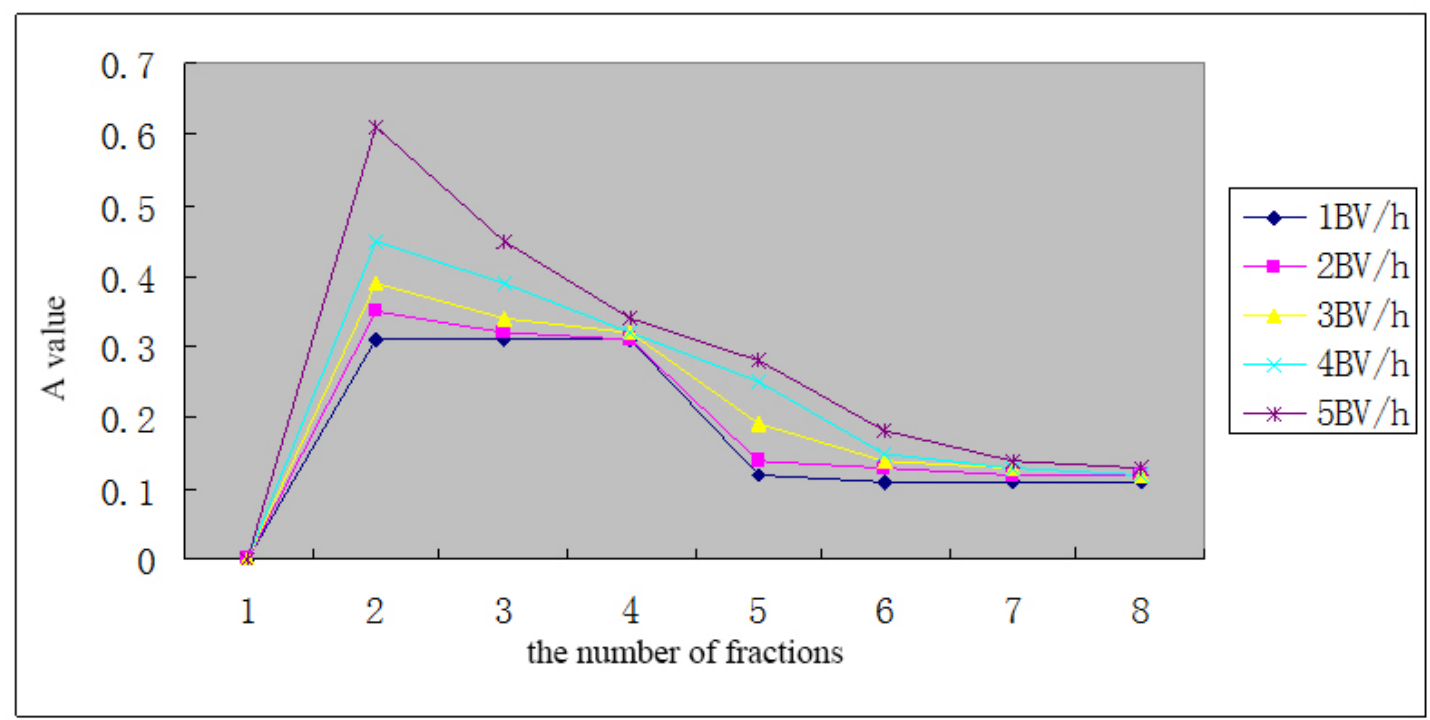


Figure 2: Investigation on adsorption effect of total astragalosides at different elution flow rates

The results showed that the resin had larger adsorption capacity at lower flow rates, adsorption capacity of resin showed a decreasing trend with the increase of flow rate, after comprehensive consideration of various factors, a flow rate of $5 \mathrm{BV} / \mathrm{h}$ was selected in the experiment.

\section{Selection of eluent}

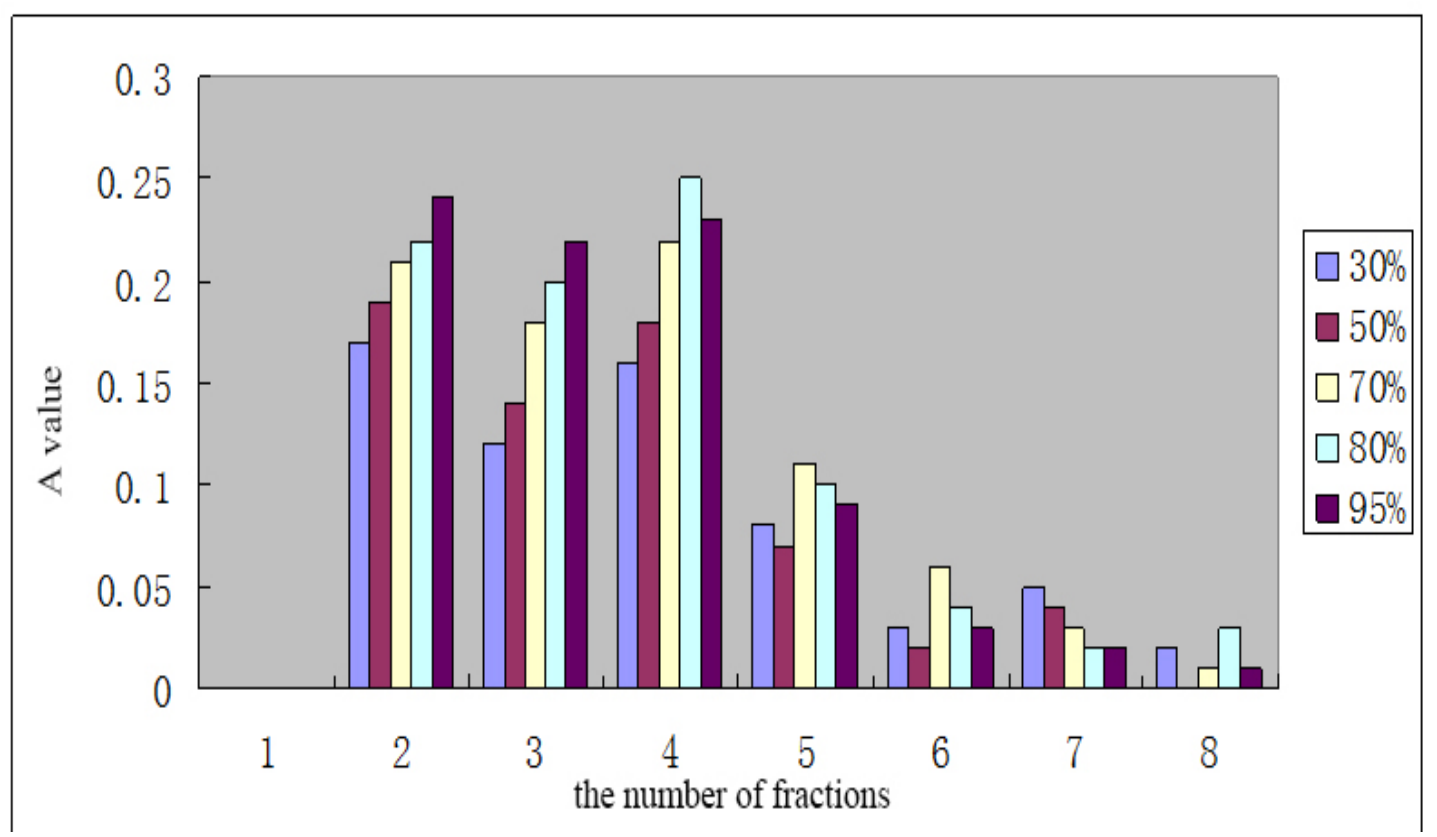

Figure 3: Investigation of elution flow rate

The experimental results showed that the desorption rate of total astragalosides increased with the increase of ethanol concentration, elution effect was the best when $80 \%$ and $95 \%$ ethanol were used as the eluent, taking into account the cost savings, $80 \%$ ethanol was selected as the eluent finally.

\section{Result of astragelosides on cell proliferation}

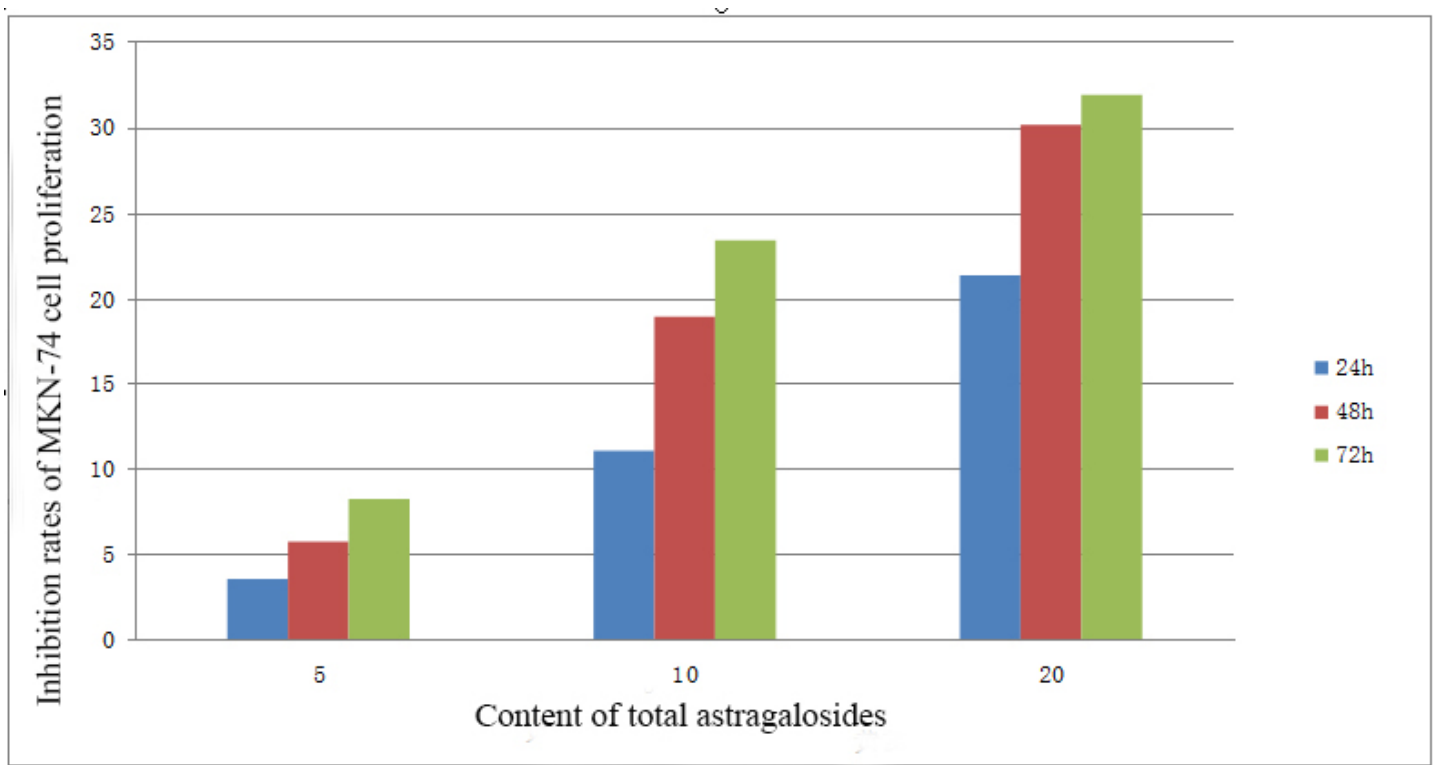


Figure 4: Inhibition rates of different concentrations of total astragelosides on MKN-74 cell proliferation

As can be seen from the experimental results, MT'T assay found that astragalosides could relatively pronouncedly inhibit the proliferation of MKN-74 cells, and the inhibitory effect was enhanced with the increase of astragaloside dose and the extension of processing time, which showed a dose-and time-dependence.

\section{Discussion}

Macroporous adsorption resin is a new type of highmolecular polymer with large pore size, and relatively large specific surface area. ${ }^{8-9}$ Owing to its high stability, high adsorption selectivity, easy regeneration, as well as environmental protection and energy conservation advantages, it has been widely used in the extraction, isolation, purification and enrichment of effective parts and monomer chemical compositions in traditional Chinese medicines and natural medicines. ${ }^{10-11}$

In this experiment, the static adsorption effects of different types of resins were determined with adsorption capacity of astragalosides as the evaluation indicator. After examining the adsorption performance of three resins, namely DA201, D101 and AB-8 macroporous adsorption resins, it was confirmed that DA201 adsorption resin had more superior adsorption performance, so DA201 adsorption resin was selected for the experiment.

In this paper, the dynamic adsorption performance of DA201 adsorption resin was examined, and the dynamic adsorption curve of total astragalosides on DA201 resin column was plotted. Meanwhile, eluent and elution flow rate were investigated, the results showed that the choice of eluent of $80 \%$ ethanol, and a flow rate of $5 \mathrm{BV} / \mathrm{h}$ could maximize the yield of total astragalosides.

In addition, this paper investigated the inhibition of human gastric cancer MKN-74 cell growth by total astragalosides, and determined the inhibitory effect of total astragalosides on proliferation of human gastric cancer MKN-74 cells by MTT assay. As can be seen from the experimental results, the M'T'T assay found that astragalosides could relatively pronouncedly inhibit the proliferation of MKN-74 cells, and the inhibitory effect was enhanced with the increase of astragaloside dose and the extension of processing time, which showed a doseand time-dependence.
It is obvious that the results of this study are as follows: The chemical methods can be good for the enrichment and purification of astragaloside, and the extraction has significantly inhibition on human gastric cancer cell MKN-74. Discovery new pharmacological activity of astragaloside is exciting, but the present study has several shortcomings and deficiencies. Such as this study did not conduct in-depth study on the mechanisms, it could not be explained clearly without the mechanism. In order to further improve the research, we decided to make anticancer mechanism of strageloside as a research priority. And we hope that it can eventually be replicated in clinical aspects and applications.

\section{References}

1. Chinese Pharmacopoeia Commission. Pharmacopoeia of the People's Republic of China: 2010 Edition Vol. 1. Beijing: China Medical Science Press. 2010; 283284.

2. Huang SW, Wu ZH. Study on Separation, Purification of Astragaloside by Macroporous Adsorption Resin. Journal of Chinese Medicinal Materials. 2006; 29(12): 1352-1355.

3. Liu JL, Wang H, Fu SP, Jin FX, Yu HS. Purification of astragalosides from biotransformates with macroporous resin. Journal of Dalian Institute of Light Industry. 2007; 26(2): 128-131.

4. Liu MH, Ren MP, Chen JP, Sun YH, Li H, Zhang Z, Xiao SH. Study on antitumor activity of Astragalus saponins. Pharmacology and Clinics of Chinese Materia Medica. 2009; 25(2): 68-70.

5. Liu GL, Zhang CY, Liu XN, Li 1, Zheng XZ. Study on the anti-proliferation effect of Astragalus polysaccharides on the human gastric cancer cell line SGC7901 in vitro. China Practical Medical. 2007; 2(13): 8-9.

6. Makiko UG, Yasuhiro K, Akitsu T, Kotaro H, Naoshi D, Koji T, Takao Y, Gordon BM, Keizo I, Junken A, Hiroyuki A. Autotaxin has lysophospholipase D activity leading to tumor cell growth and motility by lysophosphatidic acid production. Cell Bio. 2002; 15(2): 227-233. 7. Zhu ZG, Yu ZL, Cai J, Chen SS, Lei LS, Wu SG. Study on the anti-tumor effect of Tupistra Chinensis Bak. extract. Journal of Chinese Medicinal Materials. 2006; 29(3): 277-279.

8. Li P. Application of macroporous adsorption resin in the study of effective constituents in Chinese herbal medicines. Tianjin Pharmacy. 2002; 14(3): 9-11.

9. Sun JH, Meng XF. Application of Macroporous Resin and the Impact Factors on Effective Ingredient of the Extraction and Purification of the Traditional Chinese Medicine. Journal of Shandong Institute of Commerce 
and Technology. 2008; 8(6): 94-96.

10. Li YC, Meng XJ, Li XH. Dynamic adsorption and desorption characteristics of HPD-600 macroporous resin to flavonoids of blueberry leaves. Food Research and Development. 2009; 30(2): 15-18.
11. Xiang DX, Li HD, Zhu YC, Luo JY. Studies on separation and purification of total flavones from Radix Puerariae by macroreticular adsorbents. Chinese Pharmacentical Journal. 2003; 38(1): 35-37. 